\title{
Design and Optimization of a Digital Microfluidic Biochip for Protein Crystallization
}

\author{
Tao $\mathrm{Xu}^{1}$ and Krishnendu Chakrabarty ${ }^{1}$ and Vamsee K. Pamula ${ }^{2}$ \\ ${ }^{1}$ Department of Electrical and Computer Engineering \\ Duke University, Durham, NC 27708, USA \\ ${ }^{2}$ Advanced Liquid Logic, Inc., Research Triangle Park, NC 27560, USA
}

\begin{abstract}
Proteins crystallization is a commonly used technique for protein analysis and subsequent drug design. It predicts the three-dimensional arrangement of the constituent amino acids, which in turn indicates the specific biological function of a protein. Protein crystallization experiments are typically carried out manually on multi-well plates in the laboratory. These experiments are slow, expensive, and error-prone. We present the design of a multi-well plate microfluidic biochip for protein crystallization; this biochip can transfer protein samples, prepare candidate solutions, and carry out crystallization automatically. To reduce the manufacturing cost of such devices, we present an efficient algorithm to generate a pin-assignment plan for the proposed design. The resulting biochip enables control of a large number of on-chip electrodes using only a small number of pins. Based on the pin-constrained chip design, we present an efficient shuttle-passenger-like droplet manipulation method to achieve high-throughput and defect-tolerant well loading.
\end{abstract}

\section{Introduction}

Proteins play a key role in all biological processes. The specific biological function of a protein is determined by the three-dimensional (3D) arrangement of the constituent amino acids. Therefore, the 3D structure of a protein needs to be understood for effective protein engineering, bioseparations, rational drug design, controlled drug delivery, as well as the design of novel enzyme substrates, activators, and inhibitors [1]. A widely used method to study the $3 \mathrm{D}$ structure of proteins is to crystallize the proteins and determine the structure using X-ray diffraction [2].

Proteins are crystallized in mainly four different ways: batch, vapor diffusion, liquid/liquid (free interface) diffusion, and dialysis methods [3]. We focus here on batch crystallization methods, where the protein to be crystallized is mixed with the crystallizing agents at the required concentration at the start of the experiment. In this case, supersaturation is reached immediately upon mixing. Protein crystallization is a multi-parametric process that involves the steps of nucleation and growth, where molecules are brought into a thermodynamically unstable and a supersaturated state. In order to "hit" upon the correct parameters for the crystallization of proteins, a large number of experiments $\left(10^{3}\right.$ to $\left.10^{4}\right)$ are typically required, which leads to the consumption of substantial protein volumes and long time durations.

Efforts are ongoing to reduce the consumption of proteins by miniaturizing the crystallization setup. Screening for protein crystallization includes many repetitive and reproducible pipetting operations. To ease this manual and time-consuming task, several automatic methods have been introduced [4, 5]. Despite such efforts at reducing protein volumes, these processes still consume a significant amount of protein samples (in the microliter range) and they are labor-intensive.

Recent studies have focused on the application of a high-throughput and inexpensive technology, referred to as digital

*This work was supported in part by the National Institute of General Medical Sciences of the National Institute of Health (grant \# R44GM072155) and the National Science Foundation (grant \# CCF-0541055). microfluidics, to protein assays. Digital microfluidics is an emerging technology that aims to integrate fluid-handling on a chip. Bioassay protocols are scaled down (in terms of liquid volumes and assay times), and run on a microfluidic chip by manipulating discrete droplets of nanoliter volumes using a patterned array of electrodes [6]. By reducing the rate of sample and reagent consumption, digital microfluidic biochips enable continuous sampling and analysis for on-line, real-time, chemical and biological analysis, which make it uniquely suitable for high throughput protein crystallization [7]. Recent years have seen the emergence of computer-aided design tools for digital microfluidic biochips [8-11].

Recent studies have also shown the feasibility of carrying out protein crystallization on a digital microfluidic biochip. In [12], Srinivasan et al. presented a fabricated digital microfluidic biochip for protein stamping, which is capable of handling transportation and mixing of droplets enclosing protein samples with concentrations up to $0.01 \mathrm{mg} / \mathrm{ml}$. The implementation of the basic operations in protein crystallization clearly highlights the promise of a protein crystallization biochip that relies on digital microfluidics. However, no automated chip design technique has thus far been proposed.

In this paper, we present a prototype design of a multi-well plate biochip for protein crystallization. The chip layout consists of 96 wells for high-throughput processing. To reduce control complexity and fabrication cost, an efficient pin-assignment and control scheme is also proposed and applied to the design. In this way, a large number of on-chip electrodes can be controlled using a small number of control pins, with minimal impact on the system throughput. Based on the pin-constrained chip design, we present an efficient shuttle-passenger-like droplet manipulation method to achieve high-throughput and defect-tolerant well loading.

\section{Digital Microfluidics}

A digital microfluidic biochip utilizes the electrowetting phenomenon to manipulate and move nanoliter droplets containing biological and chemical samples on a two-dimensional electrode array [13]. A unit cell in the array includes a pair of electrodes that acts as two parallel plates. The bottom plate contains a patterned array of individually controlled electrodes, and the top plate is coated with a continuous ground electrode. A droplet rests on a hydrophobic surface over an electrode, as shown in Fig. 1. It is moved by applying a control voltage to an electrode adjacent to the droplet and, at the same time, deactivating the electrode just under the droplet. This electronic method of wettability control creates interfacial tension gradients that move the droplets to the charged electrode. Using the electrowetting phenomenon, droplets can be moved to any location on a two-dimensional array.

By varying the patterns of control voltage activation, many fluid-handling operations such as droplet merging, splitting, mixing, and dispensing can be executed in a similar manner. For example, mixing can be performed by routing two droplets to the same location and then turning them about some pivot points. The digital microfluidic platform offers the additional advantage of flexibility, 


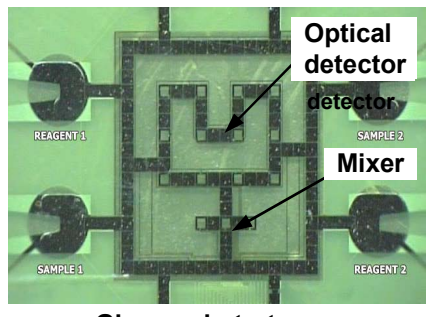

Glass-substrate

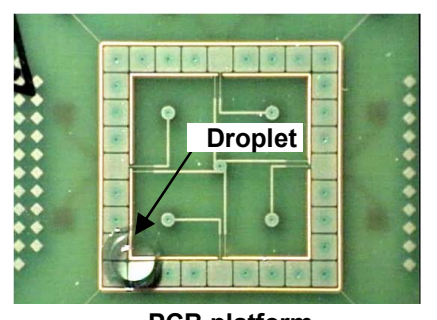

PCB platform
Fig. 1: Fabricated digital microfluidic arrays.

referred to as reconfigurability, since fluidic operations can be performed anywhere on the array. Droplet routes and operation schedules are programmed into a microcontroller that drives electrodes in the array. In addition to electrodes, optical detectors such as LEDs and photodiodes are also integrated in digital microfluidic arrays to monitor colorimetric bioassays [12]. A film (filler fluid) of silicone oil is typically used to prevent evaporation and cross contamination [13].

To address the need for low-cost, PCB technology has been employed recently to inexpensively mass-fabricate digital microfluidic biochips. Using a copper layer for the electrodes, solder mask as the insulator, and a Teflon AF coating for hydrophobicity, the microfluidic array platform can be fabricated by using an existing PCB manufacturing process [14]. This inexpensive manufacture technique allow us to build disposable PCB-based microfluidic biochips that can be easily plugged into a controller circuit board that can be programmed and powered via a standard USB port. However, a large number of independent control pins necessitates multiple PCB layers, which adds significantly to the product cost. We can address the electrodes separately by employing a serial-to-parallel interface on the device. However, this method requires active circuit components on the PCB, e.g., logic elements such as gates and flip-flops, which lead to increased cost and power consumption.

\section{Multi-Well-Plate Biochip Design for Protein Crystallization}

In this section, we present a multi-well plate design prototype for protein crystallization. As discussed in Section 1, to "hit" on the correct parameters for the crystallization of proteins, typically a very large number of experiments $\left(10^{3}-10^{4}\right)$ are required. To achieve high efficiency, we use a multi-well plate design for parallel processing, as in microbatch crystallization. The schematic for the design is shown in Fig. 2. The overall chip size is the same as that of a standard Society for Biomolecular Screening (SBS) multi-well plate. The chip has 96 wells and there are electrode pathways to connect these wells to reagent-loading and protein-loading ports.

Fig. 3 shows the specific configuration of the wells. Note that unlike microbatch crystallization, where reagents and proteins are preloaded either manually or by robotics, here reagent and protein droplets are automatically transported along the pathways from their input loading ports to the wells. The rest of the chip real estate is used for accommodating the reagent and protein input wells. In addition to the protein reservoir that a user loads, there are two additional reservoirs that the user can load. These additional reservoirs can be loaded with any user-selected additives such as glycerol or detergents. Additives can stabilize the proteins and there are numerous reports on the use of additives to improve the quality and size of protein crystals [15]. As we gain a better understanding of scaling issues, we will increase the number of wells on-chip, since space (real estate) is available.

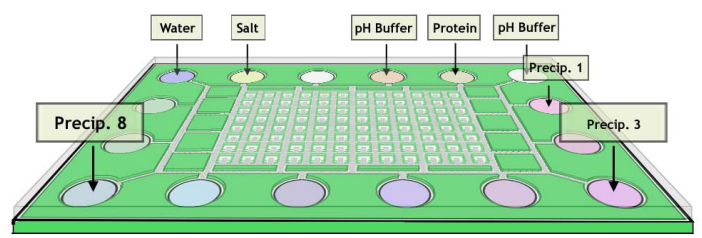

Fig. 2: Schematic view of a 96-well chip that automatically sets up 96 reagent condition solutions.

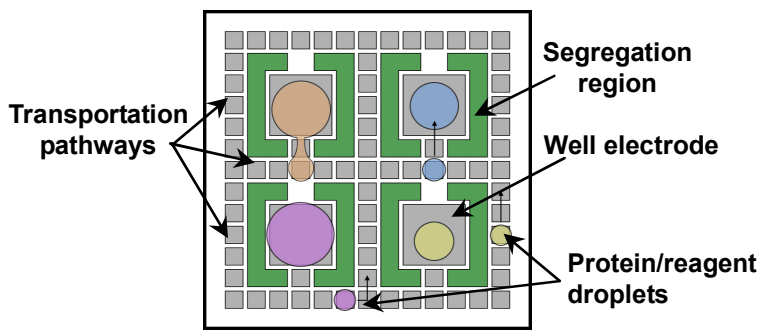

Fig. 3: Schematic top-view of four wells and the surrounding electrodes.

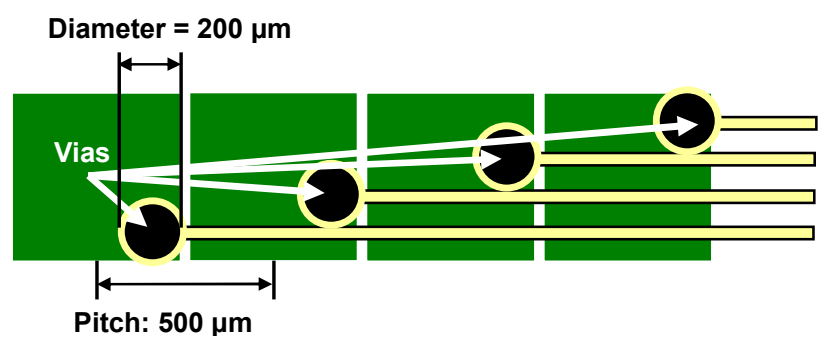

Fig. 4: Illustration of wire-routing limits on a PCB layer.

\section{Pin-Constrained Chip Design}

Next we assign control pins to address the electrodes in the proposed design. There are a total of 1284 electrodes in the chip, including electrodes in wells, transportation pathways, and reservoirs. If direct addressing is used, i.e., each cell of the patterned electrodes is accessed directly and independently via a dedicated control pin, a total of 1284 pins will need to be wired. However, a large number of electrodes leads to a cumbersome wiring problem for control pins, especially when PCB technology is used for fabrication. In PCB technology, the diameter of the via hole is usually comparable to the electrode pitch size. Therefore, there is only a limited number of control lines that can be routed on one layer of PCB. As shown in Fig. 4 , the via hole diameter is $40 \%$ of the electrode pitch. Therefore, only four control pin can be wired in any row. To route a large number of control pins, a multi-layer PCB design is needed, which is prohibitively expensive. Therefore we adopt a pin-constrained design method referred to as "Connect-5" algorithm, which allows a control pin to be connected to multiple electrodes, thereby reducing the total number of pins [16].

This pin-assignment approach relies on using a regular distribution of pins, referred to as Bagua repetition, see Fig. 5. Given a biochip array, the "Connect 5" algorithm uses tiling of the Bagua repetitions to cover all the electrodes on the array. As shown in Fig. 5, five copies of Bagua repetitions are sufficient to cover a biochip array of any size. Therefore, only 5 pins are needed to address all the electrodes on the array. The control pins assigned to the electrodes using this method in a microfluidic array allow free movement of droplets without causing unintentional operations [16].

We modify the above pin-assignment procedure above to make it applicable for our well-plate design. Note that the well-plate design 


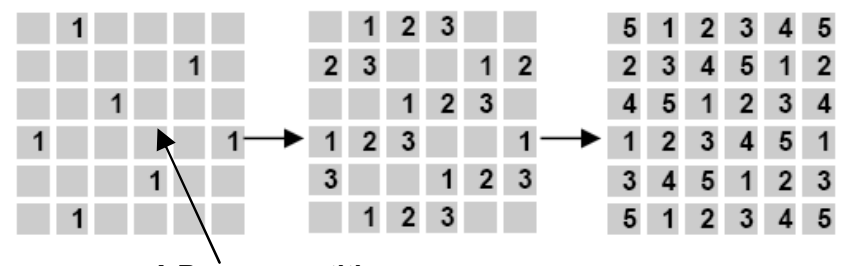

A Bagua repetition

Fig. 5: Assigning pins to an electrode array using the "Connect-5" algorithm.

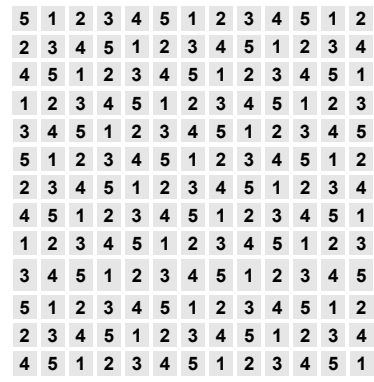

(a)

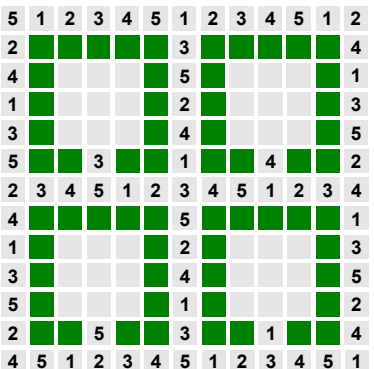

(b)

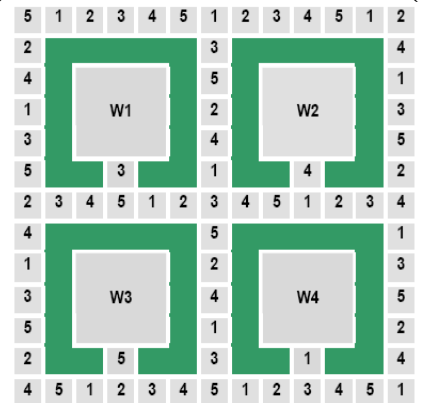

(c)

Fig. 6: Example of pin-assignment example for a 4-well-plate design.

can be viewed as a special case of the two-dimensional array where parts of the array are occupied by wells and segregation walls. Unoccupied electrodes between wells can be used as transportation pathways. Therefore, the pin-assignment for these electrodes does not need to be changed. The overall pin-assignment procedure is as follows.

1. Start with a two-dimensional electrode array of the same size as the target well-plate design, but with no cells reserved as wells or segregation regions. Apply the Connect-5 algorithm to generate a preliminary pin-assignment result. For example, to generate a pin-assignment result to the multi-well chip in Fig. 3, a preliminary result is first derived, as shown in Fig. 6(a).

2. Next, consider the electrodes that will make up the segregation regions and wells in the multi-well design. Disconnect these electrodes from their control pins, see Fig. 6(b).

3. Finally, group the electrodes occupied by each well and connect each group to a single control pin. For independent control of each well, the group control pins must be different not only from each other but also from the pins assigned to the electrodes on the transportation pathway. The modified pin-assignment result is shown in Fig. 6(c).

Note that in Fig. 6(c), the same patterns of pin assignment repeat in both column and row directions with a period of 6 . Based on this

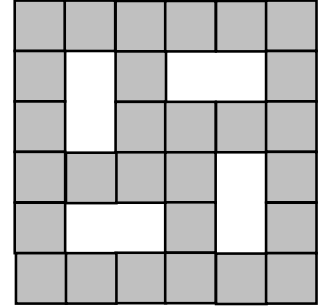

(a)

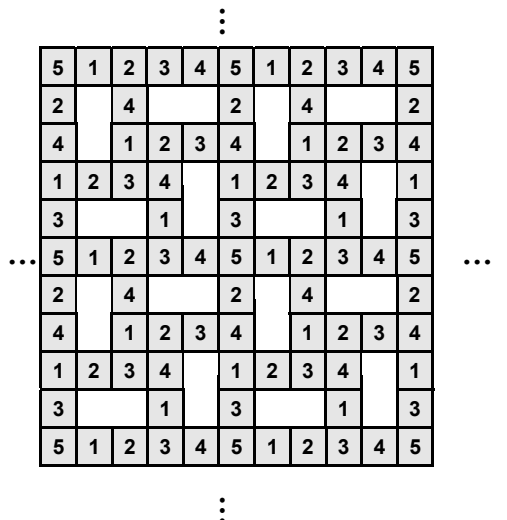

(b)

Fig. 7: (a) Illustration of a $6 \times 6$ electrode well unit. (b) Pin assignment using 5 pins for the 96-well chip (unit well size $=6 \times 6$ electrodes).

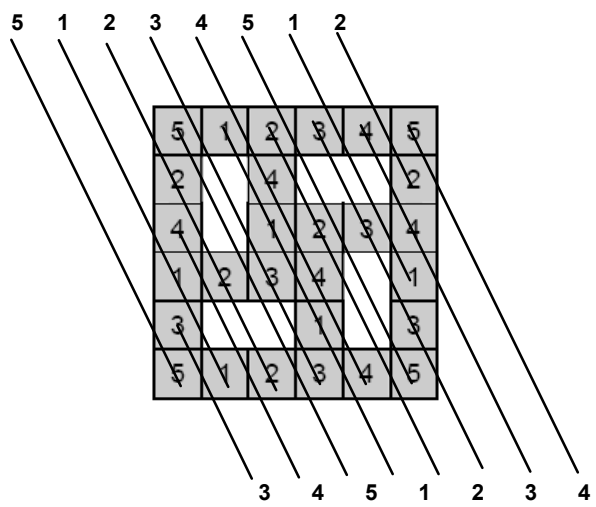

Fig. 8: Wiring of a well unit.

observation, we can adjust the size of the unit well to obtain a more regular pin-assignment result. Here define a well unit as a single well and the routing pathways round it. In the design in Fig. 6(c), the size of the well unit is $7 \times 7$. We first shrink the size of the unit well from $7 \times 7$ to $6 \times 6$ (since the period of the repetitive pin-assignment patterns is 6) electrodes, as shown in Fig. 7(a). Next we apply the Connect-5 algorithm to get a pin assignment for the 96-well chip with the adjusted unit well size, see Fig. 7(b).

For a 96 well plate design with well unit of size $6 \times 6$, there are a total of 1284 electrodes in the chip, including electrode in wells, transportation pathways and reservoirs. Therefore, a total of 1284 control pins are needed for direct addressing. In contrast, the design in Fig. 7(b) only needs 5 pins to control all the electrodes on the transportation pathways, thereby significantly reducing the total number of control pins to 181 .

The pin-constrained design using the Connect- 5 method not only significantly reduces the number of control pins but it also provides 
an easy wiring solution. According to [16], electrodes sharing the same pin in the pin-assignment result from Connect-5 algorithm are diagonally aligned. Therefore they can be easily wired diagonally, as shown in Fig. 8. Moreover, the diagonal wiring allows the diameter to be almost the same as the electrode pitch size. Thus, this efficient wiring plan allows the 181 pins to be wired on a 2-layer PCB. Recall that the direct-addressing method needs 1284 control pins, which requires a 4-layer $\mathrm{PCB}$ and thereby increases the fabrication cost by a factor of 1.6 2 [17]. Moreover, the 181 pins can be easily incorporated using standardized 3-mil feature-size technology. In contrast, to fit the 1284 pins in the direct-addressing-based design, 2 mil technology, which usually cost $3-5 x$ times more than 3 mil technology, has to be used. Therefore, the pin-constrained design achieves a reduction of fabrication cost by a factor of 5-10x. The reduction is more significant when the wiring-plan design cost is considered.

In Fig. 7(b), every well unit has the same pattern of pinassignment. This is because the dimension of the unit well is the same as the period of pin-assignment patterns form Connect-5 algorithm. This regular pin-assignment result facilitates the use of an efficient well-loading algorithm, which will be discussed in Section 5 .

\section{Shuttle-Passenger-Like Well-Loading Algorithm}

In this section, we focus on the problem of loading the wells with sample and reagent droplets on the pin-constrained chip. The goal is to efficiently route the sample and reagent droplets to their destination wells. Note that in the 96-well chip design in Fig. 7(b), every $6 \times 6$ well unit has the same pattern of pin-assignment. Therefore, any sequence of manipulations in a single well unit will cause the same manipulations in all the other well units. Although this "synchronizing" property leads to reduced freedom of droplet manipulations, it allows the concurrent manipulation of multiple droplets. Based on this observation, we propose a parallel shuttle-passenger-like routing method for high-throughput well loading.

We illustrate the well-loading algorithm using an example. Fig. 9 shows a pin-constrained chip which consists of four $6 \times 6$ well units. A dispensing reservoir is located at the top right corner on the chip. Three droplets $D_{1}, D_{2}$, and $D_{3}$ are to be dispensed and routed to three destination wells. If the droplets are placed on the start points as indicated in Fig. 11, the routing can be carried out simultaneously by applying the control-pin actuation sequence $5 \rightarrow 2 \rightarrow 4 \rightarrow 1 \rightarrow 3 \rightarrow 5 \rightarrow 4$ $\rightarrow 3 \rightarrow 2 \rightarrow 1$. The actuation sequence will route all the droplets (if any) at the upper left corner of the well units to the well within the same unit, just as synchronized shuttles that carry passengers from fixed start points to fixed destinations. The shuttles run regularly irrespective of whether there is any passenger. To go to a specific destination, a passenger needs to get to the correct starting point and wait for the shuttle (pin actuation sequence) for pick-up and routing to the destination (well).

Routing of droplets to the starting point can also be carried out using the shuttle-passenger-like method. Therefore, the proposed well-loading method contains two steps.

In the first step, droplets to be routed are transported to the corresponding start points in their destination well units. This step is carried out as follows:

a) Calculate the electrode-activation sequence to route the droplet to the farthest starting point away from the source reservoir.

b) Select a subsequence from the sequence from a) for each droplet that can be route $d$ to its starting point.

c) Applying the electrode-activation sequence from a), and dispense each droplet at a specific time corresponding to the start of its subsequence.

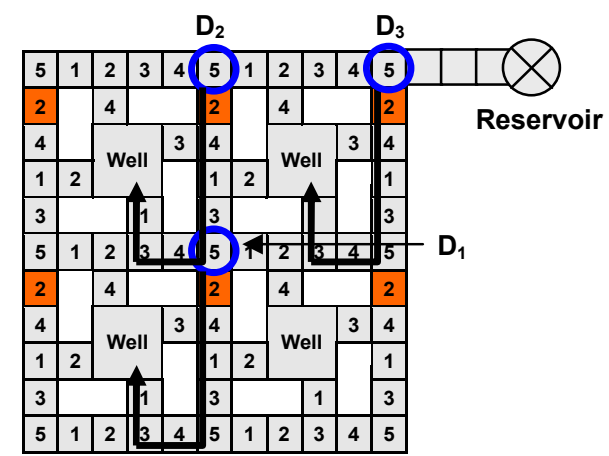

Fig. 9: Loading of three droplets using shuttle-passenger-like method.

Next, a second pin-actuation sequence is applied to route droplets to their target wells. The overall routing steps take little time because all the wells can be filled using only two pin-actuation sequences.

\section{Defect tolerance}

The design proposed in Section 4 and Section 5 may suffer from fabrication defects or operational faults. In this section, we propose a "cross loading" based method to achieve defect tolerance for the proposed chip design. We rely on the use of known testing and diagnosis methods to locate defect sites [18].

We first classify defects into three categories based on their locations on the chip. Note that the well-loading algorithm proposed in Section 5, wells are loaded from one side, i.e., right side or left side. Therefore, not all the electrodes are used. If a defect occurs in these unused electrodes, then it will not affect droplet manipulations on the chip. We refer to this type of defects as benign defects. In the design proposed in Section 4, benign defects include all the defects in the unused entrance electrodes for the well and all the electrodes between the bottom entrance electrodes and the left/right routing pathways if all the wells are loaded from the right/left side. For these benign defects, no defect tolerance is needed.

The second category of defects occurs on the electrodes used by the well-loading algorithm on the electrode rows but not on the routing pathways. These defects are referred to as loading pathway defects. They can be bypassed by simply changing the side from which the well is loaded.

The third category includes all the defects on the routing pathways. Therefore, we refer to them as routing pathway defects. Unlike loading pathway defects, these defects affect the loading operations for more than one well unit. They cannot be bypassed by simply changing the side from which the well is loaded. Instead, we use a "cross loading" method for defect tolerance. Two iterations of well-loading operations are carried out, one in the column direction and one in the row direction. If the defects occur on the routing pathways in the well-loading operation in the column direction, the loading of all the wells within the same column with the defects will be skipped. The skipped wells will then be loaded in the well-loading operation in the row direction and vice versa.

\section{Evaluation of Well-Loading Algorithm and Defect Tolerance}

In the section, we evaluate the proposed pin-constraint design and the shuttle-passenger-like well-loading algorithm.

\subsection{Loading time}

We first calculate the time needed for loading the wells on a pin-constrained chip and a chip with independent pins (direct-access). In a direct-access chip, the time required to load all 


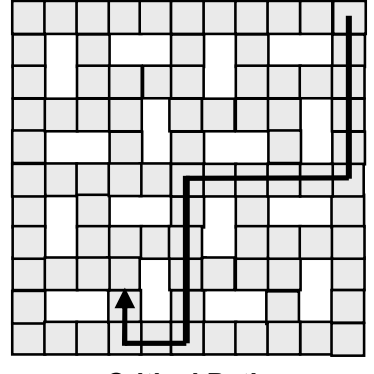

Critical Path

Fig. 10: Critical path for the multi-well chip (for both the direct-access and pin-constrained chips).

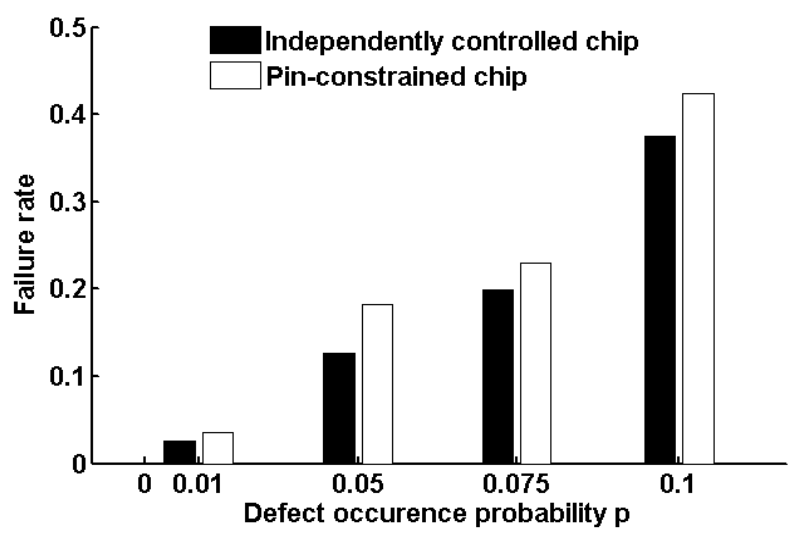

Fig. 11: Evaluation of failure rates for pin-constrained chip and independently controlled chip.

the wells is determined by the time taken by a droplet to traverse the critical path, i.e., from the dispensing reservoir to the farthest well, as shown in Fig. 10. For an $N \times N$ array, the routing time for the critical path is $2 N-3$ clock cycles. The proposed pin-constrained chip has the same critical path. Using the well loading algorithm from Section 5, a droplet can be routed along the critical path one electrode per clock cycle with no stalled cycles. Therefore, the routing time is also $2 N-3$ clock cycles. Thus we conclude that the pin-constrained design provides the same routing efficiency as the direct-access design, while it achieves a significant reduction in the number of control pins.

\subsection{Defect tolerance}

Next we examine the defect tolerance of the proposed pin-constrained design by injecting random defects. A design is deemed to be robust if the injected defect can be bypassed using the defect-tolerance methods proposed in Section 6. Some defects may block all the routing pathways to one or more wells, and these wells cannot be loaded. In this case, a failure occurs on the chip.

Next we define a parameter referred to as "failure rate". Let $N_{\mathrm{t}}$ be the total number of biochips in a representative sample, and let $N_{\mathrm{f}}$ be the number of defective chips that suffers from a failure. Then the failure rate $f$ is defined by the equation $f=N_{\mathrm{f}} / N_{\mathrm{t}}$.

We run the simulations with difference defect occurrence probabilities for the pin-constrained chip and record the failure rates. As a baseline, we also carry out defect injection for a direct-access chip. Results are obtained by averaging outcomes from 200 simulation runs, see Fig. 11. Note that if we do not set any upper limit on the well-loading time, any defect that can be bypassed in the direct-access chip can also be bypassed in the pin-constrained chip. This is because we can manipulate only one droplet to load only one well in any iteration of shuttle-passenger-like routing, which allows the same degree of freedom as in the direct-access chip. However, this scheme results in a significant increase in the well-loading time. Therefore, in our evaluation, we use a restricted definition of failure for the pin-constrained design; it refers to the case that the injected defects cannot be bypassed using the "cross loading" method.

Fig. 11 shows that, as expected, the introduction of pin constraints leads to a slightly higher failure rate compared to the direct-access chip. However, this increase is acceptable in practice due to the significant reduction in the number of control pins for the proposed design.

\section{Conclusion}

We have presented a multi-well plate based digital microfluidic biochip design for protein crystallization. The proposed biochip is capable of concurrently setting up 96 conditions, thereby achieves high throughput. We have also applied an efficient algorithm to generate a pin-assignment plan for the proposed design, which enables control of the biochip with only a small number of pins. Compared to directly addressable biochip, the proposed pin-constrained design achieves a significant reduction in fabrication cost. We have also described efficient droplet-routing algorithms for defect-tolerant well-loading.

\section{References}

[1] A. Fersht, Structure and Mechanism in Protein Science: A Guide to Enzyme Catalysis and Protein Folding. W. H. Freeman, NY, 1998.

[2] J. C. Kendrew et al., "A three-dimensional model of the myoglobin molecule obtained by x-ray analysis", Nature, vol. 181, pp. 662-666, 1958.

[3] N. E. Chayen, "Recent advances in methodology for the crystallization of biological macromolecules", J. Crystal Growth, vol. 198, pp. 649-655, 1999.

[4] N. E. Chayen et al., "An automated system for micro-batch protein crystallization and screening", J. Applied Crystallogr., vol. 23, pp. 297-302, 1990.

[5] J. R. Luft et al., "Microbatch macromolecular crystallization in micropipettes," J. Crystal Growth, vol. 196, pp. 450-455, 1999.

[6] R. B. Fair, "Digital microfluidics: is a true lab-on-a-chip possible?", Microfluidics and Nanofluidics, vol. 3, pp. 245-281, 2007.

[7] R. B. Fair et al., "Chemical and biological applications of digital-microfluidic devices". IEEE Design \& Test of Computers, vol. 24, issue 1, pp. 10-24, 2007.

[8] K. F. Böhringer, "Modeling and controlling parallel tasks in droplet-based microfluidic systems", IEEE Trans. CAD, vol. 25, pp. 329-339, 2006.

[9] E. J. Griffith et al., "Performance characterization of a reconfigurable planar array digital microfluidic system", IEEE Trans. CAD, vol. 25, pp. 340-352, 2006.

[10] T. $\mathrm{Xu}$ and K. Chakrabarty, "Integrated droplet routing in the synthesis of microfluidic biochips", Proc. DAC, pp. 948-953, 2007.

[11] P. -H. Yuh et al.. "Placement of defect-tolerant digital microfluidic biochips using the T-tree formulation", ACM Journal on Emerging Technologies in Computer Systems, vol. 3, article 3, 2007.

[12] V. Srinivasan et al., "An integrated digital microfluidic lab-on-a-chip for clinical diagnostics on human physiological fluids", Lab on a Chip, vol. 4, pp. 310-315, 2004.

[13] M. G. Pollack et al., "Electrowetting-based actuation of liquid droplets for microfluidic applications", Applied Physics Letters, vol. 77, pp. $1725-1726,2000$.

[14] J. Gong and C. J. Kim, "Two-dimensional digital microfluidic system by multi-layer printed circuit board", Proc. IEEE MEMS, 2005.

[15] A. Moreno et al., "Combination of oils and gels for enhancing the growth of protein crystals", J. Appl. Crystallogr., vol. 35, pp. 140 - 142, 2002.

[16] T. Xu et al., "Automated design of pin-constrained digital microfluidic biochips under droplet-interference constraints", ACM Journal on Emerging Technologies in Computing Systems, vol. 3, article 14, 2007.

[17] http://www.pcbdesign.org/pcb-layout/understanding-pcb-layers.

[18] T. Xu and K. Chakrabarty, "Parallel scan-like test and multiple-defect diagnosis for digital microfluidic biochips", IEEE Trans. Biomedical Circuits and Systems, vol. 1, pp. 148-158, 2007. 